\title{
STRONG CONVERGENCE OF AN IMPLICIT ITERATIVE PROCESS FOR AN INFINITE FAMILY OF STRICT PSEUDOCONTRACTIONS
}

\author{
Yeol Je Cho, Shin Min Kang, and Xiaolong Qin
}

\begin{abstract}
In this paper, we consider an implicit iterative process with errors for an infinite family of strict pseudocontractions. Strong convergence theorems are established in the framework of Banach spaces. The results presented in this paper improve and extend the recent ones announced by many others.
\end{abstract}

\section{Introduction and preliminaries}

Throughout this paper, we assume that $E$ is a real Banach space, $E^{*}$ is the dual space of $E$ and $J: E \rightarrow 2^{E^{*}}$ is the normalized duality mapping defined by

$$
J(x)=\left\{f \in E^{*}:\langle x, f\rangle=\|x\|^{2}=\|f\|^{2},\|f\|=\|x\|\right\}, \quad \forall x \in E,
$$

where $\langle\cdot, \cdot\rangle$ denotes the duality pairing between $E$ and $E^{*}$. The single-valued normalized duality mapping is denoted by $j$.

Let $T: D(T) \rightarrow R(T)$ be a nonlinear mapping, where $D(T)$ and $R(T)$ denote the domain $D(T)$ and the range $R(T)$ of $T$, respectively. In this paper, we use $F(T)$ to denote the set of fixed points of $T$.

Recall that the mapping $T$ is said to be nonexpansive if

$$
\|T x-T y\| \leq\|x-y\|, \quad \forall x, y \in D(T) .
$$

$T$ is said to be $\lambda$-strictly pseudocontractive in the terminology of BrowderPetryshyn [1] if there exists a constant $\lambda \in(0,1)$ such that

$$
\langle T x-T y, j(x-y)\rangle \leq\|x-y\|^{2}-\lambda\|x-y-(T x-T y)\|^{2}
$$

for all $x, y \in D(T)$, where $j(x-y) \in J(x-y)$.

Received April 24, 2009; Revised May 27, 2009.

2000 Mathematics Subject Classification. 47H09, 47H10, 47J25.

Key words and phrases. implicit iterative process, strict pseudocontraction, nonexpansive mapping, common fixed point, Banach space.

This work was supported by the Korea Research Foundation Grant funded by the Korean Government (KRF-2008-313-C00050). 
$T$ is said to be strongly pseudo-contractive if there exists a constant $k \in(0,1)$ such that there exists $j(x-y) \in J(x-y)$ such that

$$
\langle T x-T y, j(x-y)\rangle \leq k\|x-y\|^{2}, \quad \forall x, y \in D(T) .
$$

In 1974, Deimling [4] proved the following fixed point theorem for strong pseudo-contractions.

Theorem D. Let $E$ be a real Banach space and $C$ be a nonempty closed convex subset of $E$. Let $T: C \rightarrow C$ be a continuous strongly pseudo-contractive mapping. Then $T$ has a unique fixed point in $C$.

Concerning the convergence problem of implicit iterative processes to approximating a common fixed point for a finite family of nonexpansive mappings and strict pseudocontractions in the setting of Hilbert spaces or Banach spaces have been considered by several authors; see, for example, [2], [3], [5]-[9], [11]-[14].

In 2001, Xu and Ori [12] introduced the following implicit iteration process for a finite family of nonexpansive mappings $\left\{T_{1}, T_{2}, \ldots, T_{N}\right\}$ with a real sequence $\left\{\alpha_{n}\right\}$ in $(0,1)$ and an initial point $x_{0} \in C$ :

$$
\left\{\begin{array}{l}
x_{1}=\alpha_{1} x_{0}+\left(1-\alpha_{1}\right) T_{1} x_{1}, \\
x_{2}=\alpha_{2} x_{1}+\left(1-\alpha_{2}\right) T_{2} x_{2}, \\
\quad \cdots \\
x_{N}=\alpha_{N} x_{N-1}+\left(1-\alpha_{N}\right) T_{N} x_{N} \\
x_{N+1}=\alpha_{N+1} x_{N}+\left(1-\alpha_{N+1}\right) T_{1} x_{N+1}, \\
\quad \ldots
\end{array}\right.
$$

which can be written in the following compact form:

$$
x_{n}=\alpha_{n} x_{n-1}+\left(1-\alpha_{n}\right) T_{n} x_{n}, \quad \forall n \geq 1,
$$

where $T_{n}=T_{n}(\bmod N)$ (here, the $\bmod N$ takes values in $\left.\{1,2, \ldots, N\}\right)$. They considered the implicit iterative process for a finite family of nonexpansive mappings in the framework of Hilbert spaces.

In 2004, Osilike [5] further improved the results of $\mathrm{Xu}$ and Ori [12] from nonexpansive mappings to strict pseudo-contractions. To be more precise, he proved the following theorem.

Theorem O. Let $E$ be a real Banach space and $C$ be a nonempty closed convex subset of $E$. Let $\left\{T_{i}\right\}$ be $N$ strictly pseudocontractive self-maps of $C$ such that $F=\cap_{i=1}^{N} F\left(T_{i}\right) \neq \emptyset$, and let $\left\{\alpha_{n}\right\}$ be a real sequence satisfying the conditions:

(a) $0<\alpha_{n}<1$;

(b) $\sum_{n=1}^{\infty}\left(1-\alpha_{n}\right)=\infty$;

(c) $\sum_{n=1}^{\infty}\left(1-\alpha_{n}\right)^{2}<\infty$.

Let $x_{0} \in C$ and $\left\{x_{n}\right\}$ be the sequence defined by (1.2). Then

(1) The limit $\lim _{n \rightarrow \infty}\left\|x_{n}-p\right\|$ exists for any $p \in F$. 
(2) $\lim _{n \rightarrow \infty}\left\|x_{n}-T_{n} x_{n}\right\|=0$.

(3) $\left\{x_{n}\right\}$ converges strongly to a common fixed point of the mappings $\left\{T_{i}\right\}_{i=1}^{N}$ if and only if

$$
\begin{aligned}
& \qquad \liminf _{n \rightarrow \infty} d\left(x_{n}, F\right)=0, \\
& \text { where } d\left(x_{n}, F\right)=\inf _{p \in F}\left\|x_{n}-p\right\| .
\end{aligned}
$$

We remark that, from the view of computation, the implicit iterative scheme (1.2) if often impractical since, for each step, we must solve a nonlinear operator equation. Therefore, one of the interesting and important problems in the theory of implicit iterative algorithm is to consider the iterative algorithm with errors. That is an efficient iterative algorithm to compute approximately fixed point of nonlinear mappings. In this paper, we consider the following implicit iteration process with errors for an infinite family of strict pseudocontractions:

$$
x_{0} \in C, \quad x_{n}=\alpha_{n} x_{n-1}+\beta_{n} T_{n} x_{n}+\gamma_{n} u_{n}, \quad \forall n \geq 1,
$$

where $C$ is a nonempty closed convex subset of a real Banach space $E,\left\{T_{n}\right\}_{n=1}^{\infty}$ : $C \rightarrow C$ is an infinite family of strict pseudocontractions, $\left\{\alpha_{n}\right\},\left\{\beta_{n}\right\}$ and $\left\{\gamma_{n}\right\}$ are sequences in $[0,1]$ such that $\alpha_{n}+\beta_{n}+\gamma_{n}=1$ and $\left\{u_{n}\right\}$ is a bounded sequences in $C$.

The purpose of this paper is to prove the strong convergence of the implicit iterative process (1.3) for an infinite family of strict pseudocontractions in an arbitrary real Banach space. The results presented in the paper generalize and improve the results in Osilike [5], Xu and Ori [12] and some others.

In order to prove the main results of this paper, we also need the following lemmas.

Lemma 1.1. Let $E$ be a real Banach space and $C$ be a nonempty closed and convex subset of $E$. Let $T: C \rightarrow C$ be a $\lambda$-strict pseudocontraction. Then $T$ is $L$-Lipschitz continuous, where $L=1+\frac{1}{\lambda}$.

Proof. From (1.1), we have

$$
\lambda \|(I-T) x-(I-T) y) \|^{2} \leq\langle(I-T) x-(I-T y), j(x-y)\rangle .
$$

It follows that

$$
\lambda \|(I-T) x-(I-T) y)\left\|^{2} \leq\right\| x-y-(T x-T y)\|\| x-y \| .
$$

Therefore, we have

$$
\lambda \|(I-T) x-(I-T) y)\|\leq\| x-y \| .
$$

On the other hand, we have

$$
\lambda(\|T x-T y\|-\|x-y\|) \leq \lambda \|(I-T) x-(I-T) y) \|,
$$

which implies that

$$
\|T x-T y\| \leq L\|x-y\|,
$$

where $L=1+\frac{1}{\lambda}$. This completes the proof. 
Lemma 1.2. Let $E$ be a real Banach space and $C$ be a nonempty closed convex subset of $E$. Let $T_{n}: C \rightarrow C$ be a $\lambda_{n}$-strict pseudocontraction for each $n \geq 1$. Put $\lambda=\inf _{n \geq 1} \lambda_{n}>0$. Then we have the following:

(1) $T_{n}$ is a $\lambda$-strict pseudocontractions for each $n \geq 1$.

(2) $T_{n}$ is L-Lipschitz continuous, where $L=1+\frac{1}{\lambda}$.

(3) If $F=\bigcap_{n=1}^{\infty} F\left(T_{n}\right)$, then $F$ is a closed set in $C$.

Proof. (1) For each $n=1,2, \ldots$, since $T_{n}$ is a $\lambda_{n}$-strict pseudocontraction, we have

$$
\begin{aligned}
\left\langle T_{n} x-T_{n} y, j(x-y)\right\rangle & \leq\|x-y\|^{2}-\lambda_{n}\left\|x-y-\left(T_{n} x-T_{n} y\right)\right\|^{2} \\
& \leq\|x-y\|^{2}-\lambda\left\|x-y-\left(T_{n} x-T_{n} y\right)\right\|^{2}
\end{aligned}
$$

for all $x, y \in C$, where $j(x-y) \in J(x-y)$.

(2) From Lemma 1.1, for each $n=1,2, \ldots$, we have

$$
\left\|T_{n} x-T_{n} y\right\| \leq L_{n}\|x-y\| \leq L\|x-y\|, \quad \forall n \geq 1,
$$

where $L_{n}=1+\frac{1}{\lambda_{n}}$ and $L=1+\frac{1}{\lambda}$.

(3) Let $\left\{x_{n}^{*}\right\} \subset{ }^{\prime} F$ be any sequence such that $\left\{x_{n}^{*}\right\}$ converges strong to a point $x^{*}$. Next, we show that $x^{*} \in F$. Indeed, for any $i=1,2, \ldots$, from (2) of Lemma 1.2 , we see that

$$
\begin{aligned}
\left\|x^{*}-T_{i} x^{*}\right\| & \leq\left\|x^{*}-x_{n}^{*}\right\|+\left\|x_{n}^{*}-T_{i} x^{*}\right\| \\
& =\left\|x^{*}-x_{n}^{*}\right\|+\left\|T_{i} x_{n}^{*}-T_{i} x^{*}\right\| \\
& \leq(1+L)\left\|x^{*}-x_{n}\right\|,
\end{aligned}
$$

which implies that $x^{*} \in F\left(T_{i}\right)$ for all $i \geq 1$. That is, $x^{*} \in F$. This completes the proof.

Next, we show that the implicit iterative process (1.3) do can be employed to approximating common fixed points of an infinite family of strict pseudocontractions. Indeed, for any $x_{n-1}, u_{n} \in C$, define each mapping $W_{n}: C \rightarrow C$ by

$$
W_{n} x=\alpha_{n} x_{n-1}+\beta_{n} T_{n} x+\gamma_{n} u_{n}, \quad \forall n \geq 1 .
$$

For any $x, y \in C$, we have

$$
\left\langle W_{n} x-W_{n} y, j(x-y)\right\rangle=\beta_{n}\left\langle T_{n} x-T_{n} y, j(x-y)\right\rangle \leq \beta_{n}\|x-y\|^{2} .
$$

That is, $W_{n}$ is a strong pseudo-contraction for each $n \geq 1$. On the other hand, we have

$$
\begin{aligned}
\left\|W_{n} x-W_{n} y\right\| & =\left\|\left[\alpha_{n} x_{n-1}+\beta_{n} T_{n} x+\gamma_{n} u_{n}\right]-\left[\alpha_{n} x_{n-1}+\beta_{n} T_{n} y+\gamma_{n} u_{n}\right]\right\| \\
& =\beta_{n}\left\|T_{n} x-T_{n} y\right\| \\
& \leq \beta L\|x-y\| \\
& \leq L\|x-y\|
\end{aligned}
$$


where $L=1+\frac{1}{\lambda}$. This shows that $W_{n}$ is Lipschitz continuous for each $n \geq 1$. By Theorem $\mathrm{D}$, we see that there exists a unique fixed point $x_{n} \in C$ such that

$$
x_{n}=W_{n} x_{n}=\alpha_{n} x_{n-1}+\beta_{n} T_{n} x_{n}+\gamma_{n} u_{n}, \quad \forall n \geq 1 .
$$

That is, the implicit iterative process (1.3) is well-defined.

Lemma 1.3. Let $E$ be a real Banach space and $J$ be the normalized duality mapping. Then, for any $x, y \in E$,

$$
\|x+y\|^{2} \leq\|x\|^{2}+2\langle y, j(x+y)\rangle, \quad \forall j(x+y) \in J(x+y) .
$$

Lemma $1.4([10])$. Let $\left\{a_{n}\right\},\left\{b_{n}\right\}$ and $\left\{c_{n}\right\}$ be three nonnegative real sequences satisfying the following condition:

$$
a_{n+1} \leq\left(1+b_{n}\right) a_{n}+c_{n}, \quad \forall n \geq n_{0},
$$

where $n_{0}$ is some nonnegative integer such that $\sum_{n=1}^{\infty} b_{n}<\infty$ and $\sum_{n=1}^{\infty} c_{n}<$ $\infty$. Then the limit $\lim _{n \rightarrow \infty} a_{n}$ exists. In addition, if there exists a subsequence $\left\{a_{n_{i}}\right\} \subset\left\{a_{n}\right\}$ such that $a_{n_{i}} \rightarrow 0$, then $a_{n} \rightarrow 0$ as $n \rightarrow \infty$.

\section{Main results}

Theorem 2.1. Let $E$ be a real Banach space and $C$ be a nonempty closed convex subset of $E$. Let $T_{n}: C \rightarrow C$ be a $\lambda_{n}$-strict pseudocontraction for each $n \geq 1$. Assume that $F=\bigcap_{n=1}^{\infty} F\left(T_{n}\right) \neq \emptyset$ and $0<\lambda=\inf _{n \geq 1} \lambda_{n}<$ 1. Let $\left\{\alpha_{n}\right\},\left\{\beta_{n}\right\}$ and $\left\{\gamma_{n}\right\}$ be sequences in $[0,1]$ satisfying the condition $\alpha_{n}+\beta_{n}+\gamma_{n}=1$ for all $n \geq 1$ and $\left\{u_{n}\right\}$ be a bounded sequence in $C$. Let $\left\{x_{n}\right\}$ be a sequence generated by (1.3). If the following conditions are satisfied:

(a) $\sum_{n=1}^{\infty} \beta_{n}=\infty$;

(b) $\sum_{n=1}^{\infty}\left(\beta_{n}^{2}+\gamma_{n}\right)<\infty$,

then we have the following:

(1) The limit $\lim _{n \rightarrow \infty}\left\|x_{n}-p\right\|$ exists for any $p \in F$.

(2) $\liminf _{n \rightarrow \infty}\left\|x_{n}-T_{n} x_{n}\right\|=0$.

(3) The sequence $\left\{x_{n}\right\}$ converges strongly to a common fixed point $\bar{x} \in F$ if and only if

$$
\liminf _{n \rightarrow \infty} d\left(x_{n}, F\right)=0
$$

where $d(x, F)$ denotes the distance from $x$ to the set $F$, i.e.,

$$
d(x, F)=\inf _{y \in F}\|x-y\| .
$$

Proof. For any $p \in F$, since $\left\{u_{n}\right\}$ is bounded, we may, without loss of generality, assume that $\left\|u_{n}-p\right\| \leq M, \forall n \geq 1$. It follows from Lemma 1.3 and the 
definition of strict pseudocontractions that

$$
\begin{aligned}
& \left\|x_{n}-p\right\|^{2} \\
= & \left\|\alpha_{n}\left(x_{n-1}-p\right)+\beta_{n}\left(T_{n} x_{n}-p\right)+\gamma_{n}\left(u_{n}-p\right)\right\|^{2} \\
\leq & \alpha_{n}^{2}\left\|x_{n-1}-p\right\|^{2}+2 \beta_{n}\left\langle T_{n} x_{n}-p, j\left(x_{n}-p\right)\right\rangle+2 \gamma_{n}\left\langle u_{n}-p, j\left(x_{n}-p\right)\right\rangle \\
\leq & \alpha_{n}^{2}\left\|x_{n-1}-p\right\|^{2}+2 \beta_{n}\left(\left\|x_{n}-p\right\|^{2}-\lambda\left\|T_{n} x_{n}-x_{n}\right\|^{2}\right)+2 \gamma_{n}\left\|u_{n}-p\right\|\left\|x_{n}-p\right\| \\
\leq & \alpha_{n}^{2}\left\|x_{n-1}-p\right\|^{2}+2 \beta_{n}\left(\left\|x_{n}-p\right\|^{2}-\lambda\left\|T_{n} x_{n}-x_{n}\right\|^{2}\right) \\
& +\gamma_{n}\left(\left\|u_{n}-p\right\|^{2}+\left\|x_{n}-p\right\|^{2}\right) \\
= & \left(1-\beta_{n}-\gamma_{n}\right)^{2}\left\|x_{n-1}-p\right\|^{2}+\left(2 \beta_{n}+\gamma_{n}\right)\left\|x_{n}-p\right\|^{2} \\
& +\gamma_{n}\left\|u_{n}-p\right\|^{2}-2 \lambda \beta_{n}\left\|T_{n} x_{n}-x_{n}\right\|^{2} \\
\leq & \left(1-\beta_{n}\right)^{2}\left\|x_{n-1}-p\right\|^{2}+\left(2 \beta_{n}+\gamma_{n}\right)\left\|x_{n}-p\right\|^{2}+\gamma_{n} M^{2}-2 \lambda \beta_{n}\left\|T_{n} x_{n}-x_{n}\right\|^{2} .
\end{aligned}
$$

From the condition (b), we have that $\left(2 \beta_{n}+\gamma_{n}\right) \rightarrow 0$ as $n \rightarrow \infty$. There exists a positive integer $n_{0}$ such that $2 \beta_{n}+\gamma_{n}<\frac{1}{2}$ for all $n \geq n_{0}$. It follows from (2.2) that

$$
\begin{aligned}
& {\left[1-\left(2 \beta_{n}+\gamma_{n}\right)\right]\left\|x_{n}-p\right\|^{2} } \\
\leq & \left(1-\beta_{n}\right)^{2}\left\|x_{n-1}-p\right\|^{2}+\gamma_{n} M^{2}-2 \lambda \beta_{n}\left\|T_{n} x_{n}-x_{n}\right\|^{2}, \quad \forall n \geq n_{0} .
\end{aligned}
$$

This implies that

$$
\begin{aligned}
& \left\|x_{n}-p\right\|^{2} \\
\leq & \frac{\left(1-\beta_{n}\right)^{2}}{1-\left(2 \beta_{n}+\gamma_{n}\right)}\left\|x_{n-1}-p\right\|^{2}+\frac{\gamma_{n} M^{2}}{1-\left(2 \beta_{n}+\gamma_{n}\right)}-\frac{2 \lambda \beta_{n}\left\|T_{n} x_{n}-x_{n}\right\|^{2}}{1-\left(2 \beta_{n}+\gamma_{n}\right)} \\
= & {\left[1+\frac{\beta_{n}^{2}+\gamma_{n}}{1-\left(2 \beta_{n}+\gamma_{n}\right)}\right]\left\|x_{n-1}-p\right\|^{2}+\frac{\gamma_{n} M^{2}}{1-\left(2 \beta_{n}+\gamma_{n}\right)}-\frac{2 \lambda \beta_{n}\left\|T_{n} x_{n}-x_{n}\right\|^{2}}{1-\left(2 \beta_{n}+\gamma_{n}\right)} } \\
\leq & {\left[1+2\left(\beta_{n}^{2}+\gamma_{n}\right)\right]\left\|x_{n-1}-p\right\|^{2}+2 \gamma_{n} M^{2}-2 \lambda \beta_{n}\left\|T_{n} x_{n}-x_{n}\right\|^{2}, \quad \forall n \geq n_{0} . }
\end{aligned}
$$

It follows that

$$
\left\|x_{n}-p\right\|^{2} \leq\left[1+2\left(\beta_{n}^{2}+\gamma_{n}\right)\right]\left\|x_{n-1}-p\right\|^{2}+2 \gamma_{n} M^{2}, \quad \forall n \geq n_{0} .
$$

Putting $b_{n}=2\left(\beta_{n}^{2}+\gamma_{n}\right)$ and $c_{n}=2 \gamma_{n} M^{2}$ for all $n \geq 1$, we see that

$$
\left\|x_{n}-p\right\|^{2} \leq\left(1+b_{n}\right)\left\|x_{n-1}-p\right\|^{2}+c_{n}, \quad \forall n \geq n_{0} .
$$

On the other hand, from the conditions (b), we have that $\sum_{n=1}^{\infty} b_{n}<\infty$ and $\sum_{n=1}^{\infty} c_{n}<\infty$. It follows from Lemma 1.4 that the $\operatorname{limit}_{n \rightarrow \infty}\left\|x_{n}-p\right\|$ exists. Hence $\left\{\left\|x_{n}-p\right\|\right\}$ is bounded. Without loss of generality, we may assume that

$$
\left\|x_{n}-p\right\| \leq B, \quad \forall n \geq 1
$$


From (2.4), we arrive at

$$
\begin{aligned}
2 \lambda \beta_{n}\left\|T_{n} x_{n}-x_{n}\right\|^{2} & \leq\left(1+b_{n}\right)\left\|x_{n-1}-p\right\|^{2}+c_{n}-\left\|x_{n}-p\right\|^{2} \\
& \leq\left\|x_{n-1}-p\right\|^{2}-\left\|x_{n}-p\right\|^{2}+b_{n}\left\|x_{n-1}-p\right\|^{2}+c_{n} \\
& \leq\left\|x_{n-1}-p\right\|^{2}-\left\|x_{n}-p\right\|^{2}+b_{n} B^{2}+c_{n}, \quad \forall n \geq n_{0} .
\end{aligned}
$$

Therefore, we have

$$
2 \lambda \sum_{n=n_{0}}^{\infty} \beta_{n}\left\|T_{n} x_{n}-x_{n}\right\|^{2} \leq\left\|x_{n_{0}-1}-p\right\|^{2}+B^{2} \sum_{n=n_{0}}^{\infty} b_{n}+\sum_{n=n_{0}}^{\infty} c_{n}<\infty .
$$

This implies that

$$
\sum_{n=1}^{\infty} \beta_{n}\left\|T_{n} x_{n}-x_{n}\right\|^{2}<\infty
$$

From the condition (a), we see that

$$
\liminf _{n \rightarrow \infty}\left\|T_{n} x_{n}-x_{n}\right\|=0 .
$$

Finally, we prove the conclusion (3). The necessity of the condition (2.1) is obvious.

Next, we prove the sufficiency of the condition (2.1). From (2.6), we have

$$
\left(d\left(x_{n}, F\right)\right)^{2} \leq\left(1+b_{n}\right)\left(d\left(x_{n-1}, F\right)\right)^{2}+c_{n}, \quad \forall n \geq n_{0} .
$$

By Lemma 1.4, the condition (2.1) and (2.9), we obtain that

$$
\lim _{n \rightarrow \infty} d\left(x_{n}, F\right)=0 \text {. }
$$

On the other hand, we see that $\left\{x_{n}\right\}$ is a Cauchy sequence in $C$. Indeed, for any $t>0$, we know that $1+t \leq \exp \{t\}$. From (2.6), we have

$$
\left\|x_{n}-p\right\|^{2} \leq \exp \left\{b_{n}\right\}\left\|x_{n-1}-p\right\|^{2}+c_{n}, \quad \forall n \geq n_{0} .
$$

Hence, for any positive integers $n, m \geq n_{0}$, from (2.10), it follows that

$$
\begin{aligned}
\left\|x_{n+m}-p\right\|^{2} \leq & \exp \left\{b_{n+m}\right\}\left\|x_{n+m-1}-p\right\|^{2}+c_{n+m} \\
\leq & \exp \left\{b_{n+m}\right\}\left(\exp \left\{b_{n+m-1}\right\}\left\|x_{n+m-2}-p\right\|^{2}+c_{n+m-1}\right)+c_{n+m} \\
= & \exp \left\{b_{n+m}+b_{n+m-1}\right\}\left\|x_{n+m-2}-p\right\|^{2} \\
& +\exp \left\{b_{n+m}\right\} c_{n+m-1}+c_{n+m} \\
\leq & \cdots \\
\leq & \exp \left\{\sum_{i=n+1}^{n+m} b_{i}\right\}\left\|x_{n}-p\right\|^{2}+\exp \left\{\sum_{i=n+2}^{n+m} b_{i}\right\} \sum_{i=n+1}^{n+m} c_{i} \\
\leq & R\left(\left\|x_{n}-p\right\|^{2}+\sum_{i=n+1}^{n+m} c_{i}\right),
\end{aligned}
$$


where $R=\exp \left\{\sum_{n=1}^{\infty} b_{n}\right\}<\infty$. Since $\lim _{n \rightarrow \infty} d\left(x_{n}, F\right)=0$ and $\sum_{i=1}^{\infty} c_{i}<$ $\infty$, for any given $\epsilon>0$, there exists a positive integer $n_{1} \geq n_{0}$ such that

$$
\left[d\left(x_{n}, F\right)\right]^{2}<\frac{\epsilon^{2}}{8(R+1)}, \quad \sum_{i=n+1}^{\infty} c_{i}<\frac{\epsilon^{2}}{4 R}, \quad \forall n \geq n_{1} .
$$

Hence, there exists $q \in F$ such that

$$
\left\|x_{n}-q\right\|^{2}<\frac{\epsilon^{2}}{4(R+1)}, \quad \forall n \geq n_{1} .
$$

Combining (2.11) with (2.12), for any $n \geq n_{1}$ and $m \geq 1$, we arrive at

$$
\begin{aligned}
\left\|x_{n+m}-x_{n}\right\|^{2} & \leq 2\left(\left\|x_{n+m}-q\right\|^{2}+\left\|x_{n}-q\right\|^{2}\right) \\
& \leq 2\left\{R\left(\left\|x_{n}-q\right\|^{2}+\sum_{i=n+1}^{\infty} c_{i}\right)+\left\|x_{n}-q\right\|^{2}\right\} \\
& =2\left\{(1+R)\left\|x_{n}-q\right\|^{2}+R \sum_{i=n+1}^{\infty} c_{i}\right\} \\
& <2\left(\frac{\epsilon^{2}}{4}+\frac{\epsilon^{2}}{4}\right) \\
& =\epsilon^{2} .
\end{aligned}
$$

This implies that the sequence $\left\{x_{n}\right\}$ is a Cauchy sequence in $C$. Let $x_{n} \rightarrow \bar{x} \in$ $C$. Since $\lim _{n \rightarrow \infty} d\left(x_{n}, F\right)=0$ and $F$ is closed, we have $\bar{x} \in F$. This completes the proof.

Remark 2.2. Theorem 2.1 improves Theorem $\mathrm{O}$ in the following respects:

(1) From a finite family of mappings to an infinite family of mappings.

(2) From the view of computation, the implicit iterative scheme (1.3), which is an efficient iterative algorithm to compute approximately fixed point of nonlinear mappings, is more practical comparing with the process (1.2) since, for each step, we do not need to solve the nonlinear operator equation exactly.

As some applications of Theorem 2.1, we can obtain the following results for an infinite family of nonexpansive mappings.

Corollary 2.3. Let $E$ be a real Banach space and $C$ be a nonempty closed convex subset of $E$. Let $T_{n}: C \rightarrow C$ be a nonexpansive mappings for each $n \geq 1$. Assume that $F=\bigcap_{n=1}^{\infty} F\left(T_{n}\right) \neq \emptyset$. Let $\left\{\alpha_{n}\right\},\left\{\beta_{n}\right\}$ and $\left\{\gamma_{n}\right\}$ be sequences in $[0,1]$ satisfying the condition $\alpha_{n}+\beta_{n}+\gamma_{n}=1$ for all $n \geq 1$ and $\left\{u_{n}\right\}$ be a bounded sequence in $C$. Let $\left\{x_{n}\right\}$ be a sequence generated by the following manner:

$$
\left\{\begin{array}{l}
x_{0} \in C, \\
x_{n}=\alpha_{n} x_{n-1}+\beta_{n} T_{n} x_{n}+\gamma_{n} u_{n}, \quad \forall n \geq 1 .
\end{array}\right.
$$

If the following conditions are satisfied: 
(a) $\sum_{n=1}^{\infty} \beta_{n}=\infty$;

(b) $\sum_{n=1}^{\infty}\left(\beta_{n}^{2}+\gamma_{n}\right)<\infty$,

then we have the following:

(1) The limit $\lim _{n \rightarrow \infty}\left\|x_{n}-p\right\|$ exists for any $p \in F$.

(2) $\liminf _{n \rightarrow \infty}\left\|x_{n}-T_{n} x_{n}\right\|=0$.

(3) The sequence $\left\{x_{n}\right\}$ converges strongly to a common fixed point $\bar{x} \in F$ if and only if

$$
\liminf _{n \rightarrow \infty} d\left(x_{n}, F\right)=0
$$

where $d(x, F)$ denotes the distance from $x$ to the set $F$, i.e.,

$$
d(x, F)=\inf _{y \in F}\|x-y\| .
$$

\section{References}

[1] F. E. Browder and W. V. Petryshyn, Construction of fixed points of nonlinear mappings in Hilbert space, J. Math. Anal. Appl. 20 (1967), 197-228.

[2] R. Chen, Y. Song, and H. Zhou, Convergence theorems for implicit iteration process for a finite family of continuous pseudocontractive mappings, J. Math. Anal. Appl. 314 (2006), no. 2, 701-709.

[3] C. E. Chidume and N. Shahzad, Strong convergence of an implicit iteration process for a finite family of nonexpansive mappings, Nonlinear Anal. 62 (2005), no. 6, 1149-1156.

[4] K. Deimling, Zeros of accretive operators, Manuscripta Math. 13 (1974), 365-374.

[5] M. O. Osilike, Implicit iteration process for common fixed points of a finite family of strictly pseudocontractive maps, J. Math. Anal. Appl. 294 (2004), no. 1, 73-81.

[6] S. Plubtieng, K. Ungchittrakool, and R. Wangkeeree, Implicit iterations of two finite families for nonexpansive mappings in Banach spaces, Numer. Funct. Anal. Optim. 28 (2007), no. 5-6, 737-749.

[7] X. Qin, Y. J. Cho, and M. Shang, Convergence analysis of implicit iterative algorithms for asymptotically nonexpansive mappings, Appl. Math. Comput. 210 (2009), no. 2, 542-550.

[8] X. Qin, Y. Su, and M. Shang, On the convergence of strictly pseudo-contractive mappings in Banach spaces, J. Prime Res. Math. 3 (2007), 154-161.

[9] N. Shahzad and H. Zegeye, Strong convergence of an implicit iteration process for a finite family of generalized asymptotically quasi-nonexpansive maps, Appl. Math. Comput. 189 (2007), no. 2, 1058-1065.

[10] K. K. Tan and H. K. Xu, Approximating fixed points of nonexpansive mappings by the Ishikawa iteration process, J. Math. Anal. Appl. 178 (1993), no. 2, 301-308.

[11] S. Thianwan and S. Suantai, Weak and strong convergence of an implicit iteration process for a finite family of nonexpansive mappings, Sci. Math. Jpn. 66 (2007), no. 1, $73-81$.

[12] H. K. Xu and R. G. Ori, An implicit iteration process for nonexpansive mappings, Numer. Funct. Anal. Optim. 22 (2001), no. 5-6, 767-773.

[13] L. C. Zeng and J. C. Yao, Implicit iteration scheme with perturbed mapping for common fixed points of a finite family of nonexpansive mappings, Nonlinear Anal. 64 (2006), no. 11, 2507-2515.

[14] H. Zhou, Convergence theorems of common fixed points for a finite family of Lipschitz pseudocontractions in Banach spaces, Nonlinear Anal. 68 (2008), no. 10, 2977-2983. 
YEOL JE CHO

Department of Mathematics Education and RINS

Gyeongsang National University

JINJU 660-701, KOREA

E-mail address: yjcho@gnu.ac.kr

SHin Min Kang

Department of Mathematics And RINS

Gyeongsang National University

JinJu 660-701, KorEA

E-mail address: smkang@gnu.ac.kr

XiAOLONG QIN

Department of Mathematics

HANGZHOU NORMAL UNIVERSITY

Hangzhou 310036, P. R. China

E-mail address: qxlxajh@163.com 